\title{
Casuística de códigos ictus atendidos por 061 ARAGóN en el período 2010-2016. Factores que influyen en los tiempos de respuesta y de acceso a la fibrinólisis
}

\author{
Marta L. Muñío-Iranzo, Juan Marta-Enguita, Javier Marta-Moreno, Ángel Gasch-Gallén, Marta Sampériz-Murillo
}

Objetivo. Estudiar los tiempos de respuesta en la atención al código ictus por unidades asistenciales del 061 ARAGÓN, analizando los factores implicados y su relación con el acceso al tratamiento fibrinolítico en la fase hiperaguda.

Pacientes y métodos. Estudio descriptivo transversal sobre la asistencia extrahospitalaria a partir del registro de casos atendidos por unidades asistenciales del 061 ARAGÓN a pacientes con código ictus durante el período 2010-2016.

Resultados. Se recogieron 1.743 pacientes con código ictus (54,6\%, varones), con una edad media de 72,83 $\pm 13,1$ años. El número de ictus atendidos en 2015 y 2016 (372 y 366, respectivamente) fue mayor que la media de 201 ictus anuales en el resto de los años. El 27,2\% de los pacientes fueron atendidos entre las 08:00 y las 11:59 h, intervalo horario con mayor frecuentación. Respecto al tiempo que se tardó en atender al paciente, la media fue de 71,93 $\pm 33,64$ minutos, con mayor tiempo de respuesta en Teruel. Cuando se analizó la influencia del intervalo horario sobre el porcentaje de casos tratados con fibrinólisis, se observó una mayor tasa de fibrinólisis cuando se activó entre las 12:00 y las 15:59 h (28,1\%).

Conclusión. En el 55,3\% de los pacientes, el tiempo de respuesta del 061 fue mayor de 60 minutos, pero este tiempo no se vio condicionado por la hora de activación. Sin embargo, sí había diferencias en el porcentaje de casos de fibrinólisis en los diferentes intervalos horarios, lo que sugiere que factores distintos al tiempo de respuesta del 061 influyen en la indicación del tratamiento fibrinolítico.

Palabras clave. Atención extrahospitalaria. Código ictus. Fibrinólisis. Ictus isquémico. Stroke mimics. Tiempo de respuesta.

\section{Introducción}

La Gerencia de Emergencias Sanitarias 061 ARAGÓN, dependiente del Servicio Aragonés de Salud, tiene como misión dar respuesta, las 24 horas del día y los 365 días del año, a las demandas de atención sanitaria urgente, movilizando en cada caso el recurso que se estime más adecuado [1].

El código ictus, tal y como lo define el documento de la Estrategia Nacional de Ictus 2008, es un sistema que permite la rápida identificación, notificación y traslado de los pacientes con ictus a los servicios de urgencias hospitalarias que sean capaces de ofrecer una evaluación especializada y terapia de reperfusión [2,3]. Se fundamenta en la existencia de protocolos de actuación compartidos y en la coordinación de todos los eslabones de la cadena asistencial al ictus. Se puso en marcha en Aragón en 2005.

Posteriormente, se integró en el Programa de Atención al Ictus en Aragón desde su aprobación en 2009 [4], que busca mejorar la calidad asistencial de los pacientes con ictus y disminuir su morbimortalidad, coordinando todos los recursos asistenciales.
Por otro lado, en Aragón, el ictus es la principal causa de muerte. En 2014, fue la primera en las mujeres, la segunda en los hombres y la primera en ambos sexos por encima de los 75 años [5]. Como en el resto de España, la mortalidad por esta causa presenta un descenso continuado en los últimos 30 años. Partiendo de unos niveles algo superiores a la media nacional, en Aragón este descenso ha sido mayor en los últimos 10 años (Fig. 1).

Cada año se atienden en nuestros hospitales unos 2.800 ictus. En concreto, en 2014 fueron 2.796, de los cuales 1.724 (40\%) eran infartos isquémicos, con una edad media de 77,3 años y una tasa de letalidad en el ingreso del 13,7\% [6].

La población en Aragón está envejecida y la densidad es muy dispar a lo largo del territorio, máxima en Zaragoza, con 55 hab. $/ \mathrm{km}^{2}$, y mínima en Teruel, con $9 \mathrm{hab} . / \mathrm{km}^{2}$, con una media baja $\left(27,5 \mathrm{hab} . / \mathrm{km}^{2}\right)$ en comparación con la densidad media en España (92 hab. $/ \mathrm{km}^{2}$ ) [7], lo que unido a las largas distancias (9\% del territorio nacional, $2,3 \%$ de la población) hace que sea esperable una menor tasa de fibrinólisis intravenosa, ya que es previsible que al-
Servicio de Neurología; Complejo Hospitalario de Navarra; Pamplona, Navarra (J. Marta-Enguita). Hospital Universitario Miguel Servet (M.L. Muñío-Iranzo, J. Marta-Moreno) Facultad de Ciencias de la Salud; Universidad de Zaragoza (A. GaschGallén). Gerencia de Urgencias y Emergencias Sanitarias 061 ARAGÓN (M. Sampériz-Murillo). Zaragoza, España.

Correspondencia:

Dra. Marta L. Muñío Iranzo. Hospital Universitario Miguel Servet. Paseo Isabel la Católica, 1-3. E-50009 Zaragoza.

E-mail:

mlmiranzo@gmail.com

Aceptado tras revisión externa: 02.07.19.

Cómo citar este artículo: Muñío-Iranzo ML, Marta-Enguita J, Marta-Moreno J, Gasch-Gallén A Sampériz-Murillo M. Casuística de códigos ictus atendidos por 061 ARAGÓN en el período 2010-2016. Factores que influyen en los tiempos de respuesta y de acceso a la fibrinólisis. Rev Neurol 2019; 69: 409-16. doi: $10.33588 / \mathrm{rn} .6910$. 2019117.

(c) 2019 Revista de Neurología 
Figura 1. Información epidemiológica básica, 2017: a) Estructura de la población por edad y sexo; b) Mortalidad por enfermedad cerebrovascular en Aragón y conjunto de España, años 2006-2017 [10].

a
Pirámide de población. Aragón Años 1991 y 2017

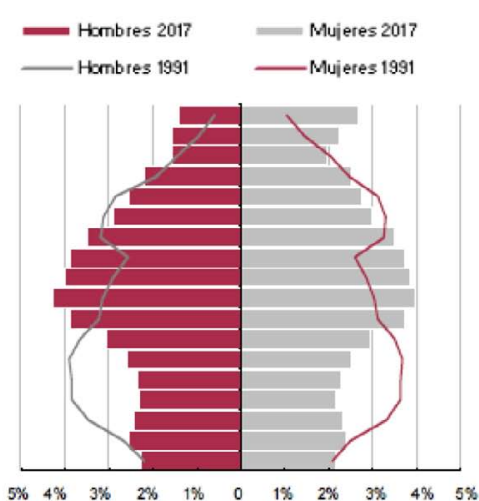

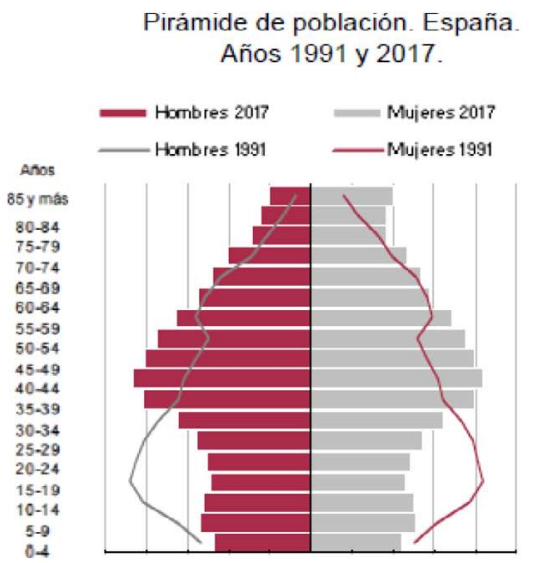

0.4 $\begin{array}{lllllllllll}5 \% & 4 \% & 3 \% & 2 \% & 1 \% & 0 & 1 \% & 2 \% & 3 \% & 4 \% & 5 \%\end{array}$

b

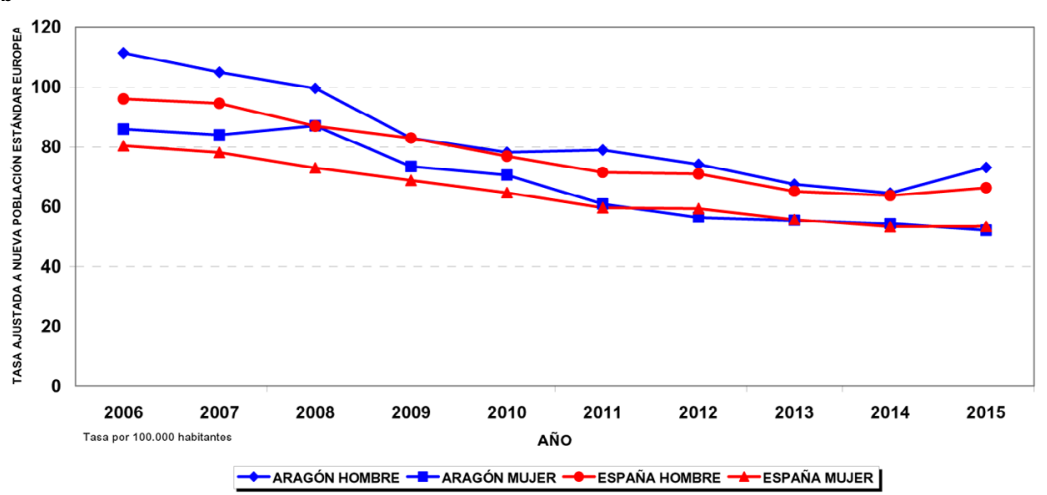

tro del período de ventana terapéutica, optimizando el tiempo hasta la reperfusión, buscando la correcta selección del caso, la eficacia y la seguridad. Esta relación entre menores tiempos y mejores resultados, en términos de menos mortalidad, menos hemorragias y más pacientes independientes en el momento del alta, se ha demostrado ampliamente en la clínica [8].

La activación del código ictus se inicia con una llamada por parte del paciente, de sus familiares o del centro de atención primaria donde se examine inicialmente al paciente. Esa llamada se recibe en el centro coordinador de urgencias, que activa por vía telefónica a la unidad asistencial que prestará la asistencia y también al centro hospitalario al cual va a derivarse al paciente en el caso de que se cumplan los criterios de traslado. Una vez que se llega al hospital, se pone en marcha otro dispositivo complementario, el código ictus intrahospitalario, que busca minimizar el tiempo hasta realizar la tomografía computarizada (tiempo puerta-TC) y seleccionar el caso para comenzar el tratamiento fibrinolítico cuanto antes (tiempo puerta-aguja). Mientras que estos tiempos intrahospitalarios han sido objeto de numerosas publicaciones, se ha prestado menos atención a los tiempos prehospitalarios. Si consideramos que el tiempo transcurrido hasta el inicio de la terapia de reperfusión es clave para los resultados, es obvio que lo serán cada uno de los tiempos intermedios, pues el tiempo final es el resultado de la suma de todos los tiempos parciales. Las guías actuales [7] enfatizan la importancia de organizar adecuadamente la asistencia prehospitalaria, pues conseguir objetivos como iniciar el tratamiento en menos de tres horas desde su comienzo o iniciar la fibrinólisis intravenosa en menos de 60 minutos tras la llegada al hospital se relacionan directamente con el uso de un código ictus bien articulado.

En este trabajo se incluyen todos los códigos ictus atendidos por unidades asistenciales del 061 ARAGÓN en el período 2010-2016, y se estudian los factores que influyen en los tiempos de respuesta y acceso a la fibrinólisis intravenosa, con especial hincapié en la variación por tramos horarios, días de la semana y estación del año.

\section{combinante (rtPA)}

El tratamiento con fibrinólisis intravenosa se indica en un período ventana de hasta 4 horas y 30 minutos desde el inicio de la clínica, el beneficio de su aplicación disminuye de forma proporcional al tiempo y las complicaciones aumentan conforme la isquemia evoluciona [7]. El objetivo del código ictus es maximizar el número de casos que llegan den-

\section{Pacientes y métodos}

Se realizó un estudio descriptivo transversal sobre la asistencia sanitaria extrahospitalaria a las personas que sufrieron un ictus y fueron atendidas por unidades asistenciales del 061 ARAGÓN. La infor- 
mación utilizada para el estudio se extrajo de las historias clínicas generadas por la asistencia 061 ARAGÓN, tras obtener el permiso de la Dirección Gerencia del 061 ARAGÓN y con el informe favorable del Comité Ético de Investigación Clínica de Aragón (PI15/0276). Se incluyeron pacientes desde el año 2010 al 2016. El estudio se llevó a cabo en la Comunidad Autónoma de Aragón, con 1.308.750 habitantes en el último censo (año 2017) [9], considerando toda la población residente en Aragón que ingresó en un hospital por un ictus, independientemente de la edad y el sexo. Las variables demográficas recogidas fueron: sexo, edad, factores de riesgo cardiovascular (diabetes mellitus, hipertensión arterial), diagnóstico final, año, mes, día de la semana, estación del año y provincia en la que el paciente sufrió el ictus. Por otro lado, las variables resultado fueron los tiempos de respuesta: tiempo de preaviso (desde que ocurre el evento hasta que entra la llamada en el centro coordinador de urgencias), tiempo 061 (desde que entra la llamada en el centro coordinador de urgencias y actúa el 061 hasta la llegada del paciente al hospital) y tiempo total (suma de los previos, desde el momento en el que el paciente comienza con el inicio de los síntomas hasta que llega al hospital). También se recogió el número de fibrinólisis intravenosas por años, y se distinguió a los que habían sido llevados al hospital por el 061 frente a los que no, así como el hospital al que se llevó al paciente. Se recogía el porcentaje de casos cuyo diagnóstico final en el momento del alta fue ictus isquémico, ictus hemorrágico, accidente isquémico transitorio y otros (crisis epiléptica, síndrome confusional y otros). En el análisis se consideraron los siguientes tramos horarios de cuatro horas: 08:00-11:59, 12:00-15.59, 16:00-19:59, 20:0023:59, 00:00-03:59 y 04:00-07:59 h.

El análisis estadístico se efectuó con el programa SPSS v. 22.0 y se realizó un análisis descriptivo de variables demográficas, temporales, de lugar y clínicas, con una descripción de frecuencias, medidas de centralidad y dispersión, con análisis de distribución de normalidad (Kolmogorov-Smirnov) cuando procede. Los resultados se dan como media \pm desviación estándar, especificándolo en otro caso. En el análisis bivariante con contraste de hipótesis usamos la comparación de proporciones cuando ambas variables sean cualitativas (chi cuadrado, prueba exacta de Fisher); las comparaciones de medias cuando una de ellas sea cuantitativa ( $t$ de Student, ANOVA y, si no siguen una distribución normal, pruebas no paramétricas, como el test $U$ de Mann-Whitney o el test de Kruskal-Wallis); y la correlación entre variables cuantitativas mediante el coe-
Tabla I. Análisis descriptivo demográfico y clínico de los 1.743 ictus incluidos.

\begin{tabular}{|c|c|c|}
\hline \multicolumn{2}{|c|}{ Edad media (años) } & \multirow{2}{*}{$\begin{array}{c}72,83 \pm 13,06 \\
951(54,6 \%)\end{array}$} \\
\hline & Varón & \\
\hline & Mujer & $787(45,3 \%)$ \\
\hline \multirow{4}{*}{ Edad } & $\leq 65$ años & $449(25,9 \%)$ \\
\hline & 66-75 años & $393(22,7 \%)$ \\
\hline & $76-85$ años & $652(37,6 \%)$ \\
\hline & $>85$ años & $238(13,7 \%)$ \\
\hline \multirow{3}{*}{ Provincia } & Huesca & $233(18 \%)$ \\
\hline & Zaragoza & $989(76,3 \%)$ \\
\hline & Teruel & $75(5,8 \%)$ \\
\hline \multirow{3}{*}{$\begin{array}{l}\text { Factores } \\
\text { de riesgo } \\
\text { cardiovascular }\end{array}$} & Hipertensión arterial & $740(42,5 \%)$ \\
\hline & Diabetes mellitus & $286(16,4 \%)$ \\
\hline & Infarto agudo de miocardio & $31(1,8 \%)$ \\
\hline \multirow{7}{*}{$\begin{array}{l}\text { Centro } \\
\text { hospitalario }\end{array}$} & Hospital Clínico Universitario & $392(30,2 \%)$ \\
\hline & Hospital Universitario Miguel Servet & $446(34,4 \%)$ \\
\hline & Hospital de Barbastro & $132(10,2 \%)$ \\
\hline & Hospital Royo Villanova & $108(8,3 \%)$ \\
\hline & Hospital San Jorge & $113(8,7 \%)$ \\
\hline & Hospital de Calatayud & $31(2,4 \%)$ \\
\hline & Hospital Obispo Polanco & $75(5,8 \%)$ \\
\hline \multirow{3}{*}{$\begin{array}{l}\text { Escala de coma } \\
\text { de Glasgow }\end{array}$} & Grave $(<8)$ & $39(4,3 \%)$ \\
\hline & Moderado (8-13) & $206(22,9 \%)$ \\
\hline & Leve (14-15) & $656(72,8 \%)$ \\
\hline
\end{tabular}

ficiente de correlación de Spearman para distribuciones no paramétricas. En todos los casos de contraste de hipótesis se consideró un intervalo de confianza al 95\% (IC 95\%).

\section{Resultados}

Se incluyó a 1.743 pacientes con activación de código ictus entre enero de 2010 y diciembre de 2016. 
Figura 2. Variables temporales y hora de activación de la unidad asistencial 061 ARAGÓN.

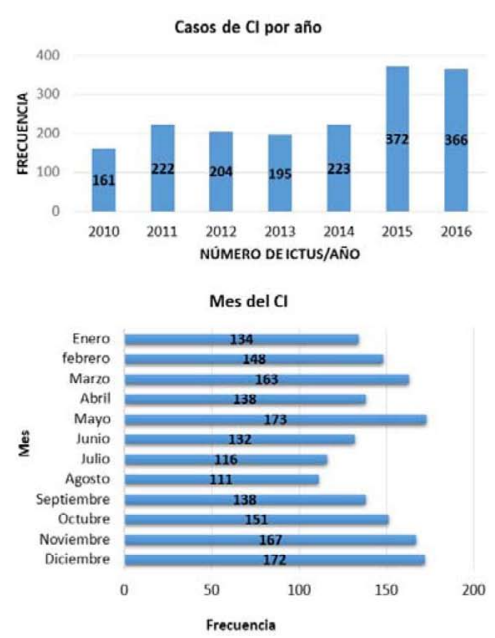

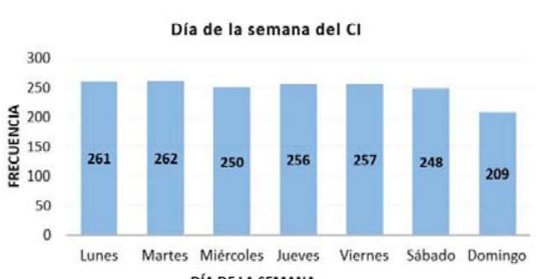
DIA DE LA SEMANA

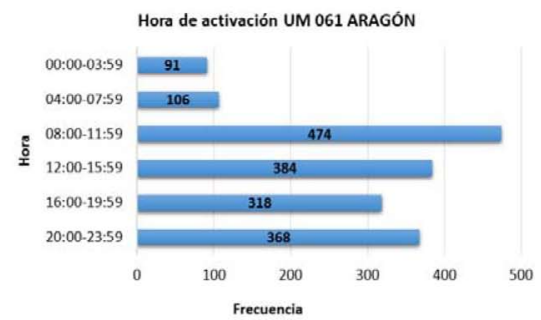

La media de edad fue de 72,83 $\pm 13,1$ años, con un $54,6 \%$ de hombres (Tabla I). En la distribución observada por años se aprecia un aumento de ictus atendidos en los años 2015 y 2016 (372 y 366, respectivamente) con respecto a la media de 201 ictus/ año en el resto de los años (Fig. 2).

Estos 1.743 códigos ictus representan el 9,3\% del total de ictus atendidos en nuestros hospitales, con una distribución anual reflejada en la tabla II.

Para considerar la proporción de ictus en pacientes jóvenes, se separaron las edades en períodos por sexo y se obtuvieron diferencias significativas. En pacientes menores de 55 años, destacó un $60,8 \%$ en los hombres frente a un $39,2 \%$ en las mujeres (Tabla III).

De los códigos ictus activados, el diagnóstico final fue de un $48,7 \%$ de infartos, $14,5 \%$ de ataques isquémicos transitorios, $19,8 \%$ de hemorragias y $16,9 \%$ de otros diagnósticos; la causa más frecuente de stroke mimics fueron las crisis epilépticas (22\%).

En el período 2010-2015 se realizaron 412 fibrinólisis intravenosas en Aragón. Por ejemplo, en 2014 se realizaron 125 fibrinólisis intravenosas, de las cuales 63 se hicieron en el Hospital Universitario Miguel Servet.

El 27,2\% de los pacientes fueron atendidos en el tramo horario entre las 08:00 y las 11:59 h. Fue el de máxima frecuentación, y entre las 00:00 y las 03:59 h fue en el que menos ictus se atendieron (5,2\%). En cuanto al día de la semana y el mes en el que fueron atendidos, no se encontraron diferencias significativas (Fig. 2).

Respecto al tiempo total (desde el evento hasta su llegada al hospital), en los atendidos por el 061 fue de 113,95 $\pm 55,16$ minutos. El tiempo 061 (el que el 061 ARAGÓN tardó en llegar al paciente) fue de $71,93 \pm 33,64$ minutos. La media del tiempo preaviso fue de $42,73 \pm 46,05$ minutos. Por sexo, los tiempos de actuación en minutos fueron los siguientes:

- Tiempo de preaviso: hombres, 44,09 $\pm 48,7 \mathrm{mi}$ nutos; mujeres, 41,18 $\pm 42,7$ minutos.

- Tiempo 061: hombres, 72,6 \pm 34,7 minutos; mujeres, $71 \pm 32,3$ minutos.

- Tiempo total: hombres, $116,4 \pm 57,6$ minutos; mujeres, $111 \pm 52,1$ minutos.

Se observó que el tiempo era ligeramente mayor en los hombres que en las mujeres, con diferencias estadísticamente significativas solamente en el tiempo total. En el análisis de tiempos, en el $55,3 \%$ de los pacientes el tiempo 061 fue mayor de $60 \mathrm{minu}$ tos (Tabla IV). En cuanto a la comparación de los tiempos por provincia, se encuentran diferencias significativas en el tiempo 061, con mayor tiempo de respuesta en Teruel con respecto a Zaragoza y Huesca. No se encontraron diferencias en los tiempos de respuesta por día de la semana ni por hora de activación, pero sí en la comparación del tiempo de preaviso según la hora de activación, en la que se encontraron diferencias significativas por tramos horarios (Tabla IV).

Cuando se analiza la relación de estas variables con el porcentaje de casos finalmente fibrinolisados, destaca que, cuando el código ictus se activó en el tramo de 12:00-15:59 h, se observó un mayor porcentaje de casos tratados $(28,1 \%)$. Sin embargo, en los períodos de activación de 20:00-23:59 y de 04:0007:59 h, se apreció una menor frecuencia de administración de rtPA (19,8\% y $16 \%$, respectivamente), con diferencias estadísticamente significativas (Tabla V).

El porcentaje de fibrinólisis intravenosa en los pacientes atendidos por el 061 es significativamente más alto que el porcentaje en los que no vienen por el 061, es decir, que vienen por otros medios (Tabla II).

\section{Discusión}

La población de estudio es el total de habitantes de Aragón, que es una población envejecida, con una tasa de recambio negativa (Fig. 1) y dispersa en un territorio amplio con densidad baja. Por comparación, el índice de envejecimiento en Aragón fue en el año 2017 de 140,25 [10], frente a los de Asturias $(209,95)[10]$, Murcia $(83,38)[10]$, Cataluña $(111,87)$ [10], Madrid $(103,76)$ [10] y la media de España 
Tabla II. Casos de ictus atendidos por el 061 ARAGÓN y fibrinólisis intravenosa (FI).

\begin{tabular}{lccccccc}
\hline Totales & FI & $\begin{array}{c}\text { Códigos ictus } \\
\text { atendidos por el 061 } \\
\text { (\% del total de ictus) }\end{array}$ & $\begin{array}{c}\text { Flatendidas } \\
\text { por el 061 }\end{array}$ & $\begin{array}{c}\text { \% de casos } \\
\text { atendidos por } \\
\text { el 061 que son FI }\end{array}$ & $\begin{array}{c}\text { \% de casos no } \\
\text { atendidos por } \\
\text { el 061 que son FI }\end{array}$ & $p$ \\
\hline 2010 & 2.807 & 75 & $161(5,73 \%)$ & $30(40 \%)$ & $18,6 \%$ & $1,7 \%$ & $<0,001$ \\
\hline 2011 & 2.778 & 112 & $222(7,99 \%)$ & $61(54,46 \%)$ & $27,5 \%$ & $2 \%$ & $<0,001$ \\
\hline 2012 & 2.777 & 114 & $204(7,34 \%)$ & $50(43,85 \%)$ & $24,5 \%$ & $2,5 \%$ & $<0,001$ \\
\hline 2013 & 2.680 & 134 & $195(7,27 \%)$ & $55(41,04 \%)$ & $28,2 \%$ & $3,2 \%$ & $<0,001$ \\
\hline 2014 & 2.438 & 125 & $223(9,14 \%)$ & $50(40 \%)$ & $22,4 \%$ & $3,4 \%$ & $<0,001$ \\
\hline 2015 & 2.631 & 168 & $370(14,06 \%)$ & $82(48,80 \%)$ & $22,2 \%$ & $3,8 \%$ & $<0,001$ \\
\hline 2016 & 2.413 & 213 & $366(15,16 \%)$ & $84(39,43 \%)$ & $23,0 \%$ & $6,3 \%$ & $<0,001$ \\
\hline $2010-2016$ & 18.524 & 941 & $1.743(9,3 \%)$ & $412(43,7 \%)$ & $23,7 \%$ & $3,5 \%$ & $<0,001$ \\
\hline
\end{tabular}

$(118,26)$ [10]. Estas características se deben tener en cuenta al analizar las variables de incidencia y los tiempos de asistencia cuando las comparamos con las de otros territorios.

En los 1.743 pacientes incluidos en el código ictus, la media de edad fue de 72,83 $\pm 13,1$ años, y se aprecian diferencias significativas respecto a otros estudios referidos a Madrid (69,24 $\pm 12,88$ años) [11] o Murcia (68,51 años; IC 95\%: 67,08-69,95 años) [12].

En este estudio se analizan los tiempos de respuesta en la atención extrahospitalaria al ictus agudo. El tiempo 061, definido como el transcurrido desde la llamada de activación al centro coordinador de urgencias y la llegada al hospital, se analiza dividido en tres intervalos: < $30 \mathrm{~min}, 30-60 \mathrm{~min}$ y $>60$ min. En el 55,3\% de los pacientes, el tiempo 061 fue superior a 60 minutos. Al comparar este resultado con el descrito en la bibliografía, se aprecia una variabilidad amplia en los formatos de recogida y en los tiempos de respuesta encontrados, como es esperable dadas las diferentes características territoriales y densidades. En el Hospital Clínico Universitario Virgen de la Arrixaca, el 36,3\% se trata en los primeros 60 minutos, mientras que en Madrid se ha publicado una mediana de 84 minutos (rango intercuartílico: 60-120 min) [11].

Un 16,9\% de los casos presentó otros diagnósticos neurológicos, y la causa más frecuente de stroke mimics fueron las crisis epilépticas (22\%); el estudio de Avellaneda-Gómez et al [13] presentó un 26\% de crisis epilépticas.

En el estudio de Okano et al [14], los stroke mimics más frecuentes fueron la epilepsia sintomática
Tabla III. Intervalos de edad por sexo $(p<0,001)$.

\begin{tabular}{lcc}
\hline & Hombres & Mujeres \\
\hline$<55$ años & $115(60,8 \%)$ & $74(39,2 \%)$ \\
\hline $56-65$ años & $172(66,7 \%)$ & $86(33,3 \%)$ \\
\hline $66-75$ años & $238(60,6 \%)$ & $155(39,4 \%)$ \\
\hline $76-85$ años & $325(49,9 \%)$ & $326(50,1 \%)$ \\
\hline$>85$ años & $97(40,9 \%)$ & $140(59,1 \%)$ \\
\hline
\end{tabular}

(20,4\%), los trastornos psiquiátricos, como la histeria y la neurosis de ansiedad (15,3\%), la hipoglucemia $(10,9 \%)$ y la disección aórtica aguda $(9,5 \%)$. Por otro lado, según Tobin et al [15], entre los stroke mimics destacaron las encefalopatías (17\%), la migraña (11\%) y el síncope (15\%).

Como es de esperar, la distribución de casos por días de la semana y por meses no es diferente. Tampoco se observan diferencias estacionales en el número de casos trasladados por el 061, aunque se observó un descenso en las activaciones del código ictus en los meses de verano (julio y agosto). En cuanto a los tiempos de respuesta, la atención extrahospitalaria por el 061 ARAGÓN no se ve influida por estos factores temporales, con tiempos de respuesta homogéneos independientemente del día de la semana o el mes del año.

Por otra parte, se encuentran diferencias significativas en los tiempos de respuesta por provincias, 
Tabla IV. Tiempos de respuesta por provincias, días de la semana y hora de activación.

\begin{tabular}{|c|c|c|c|c|c|c|c|}
\hline & & $\begin{array}{c}\text { Tiempo } \\
\text { de preaviso }\end{array}$ & $p$ & Tiempo 061 & $p$ & $\begin{array}{c}\text { Tiempo } \\
\text { total }\end{array}$ & $p$ \\
\hline & Zaragoza & $45(55)$ & \multirow{3}{*}{0,705} & $65(44,5)$ & \multirow{3}{*}{0,024} & $100(60)$ & \multirow{3}{*}{0,432} \\
\hline \multirow[t]{3}{*}{ Provincia $^{a}$} & Huesca & $45(69)$ & & $69(40)$ & & $103(59,5)$ & \\
\hline & Teruel & $43(62,8)$ & & $87(49)$ & & 115 (66) & \\
\hline & $<30 \min$ & & & $61(3,5 \%)$ & & & \\
\hline \multirow{2}{*}{$\begin{array}{l}\text { Total en } \\
\text { Aragón }{ }^{b}\end{array}$} & $30-60 \mathrm{~min}$ & & & $718(41,2 \%)$ & & & \\
\hline & $>60 \mathrm{~min}$ & & & $964(55,3 \%)$ & & & \\
\hline \multirow{6}{*}{$\begin{array}{l}\text { Hora de } \\
\text { activación }^{\text {a }}\end{array}$} & $00: 00-03: 59 \mathrm{~h}$ & 30 (15) & \multirow{6}{*}{0,039} & $69(44)$ & \multirow{6}{*}{ 0,329 } & $99(56)$ & \multirow{6}{*}{$-0,252$} \\
\hline & $04: 00-07: 59 \mathrm{~h}$ & $31,50(46,25)$ & & $67(49,25)$ & & $112(72,5)$ & \\
\hline & $08: 00-11: 59 \mathrm{~h}$ & $30(44)$ & & $65(44,25)$ & & $100(67,5)$ & \\
\hline & $12: 00-15: 59 \mathrm{~h}$ & $30(27,75)$ & & $65(44)$ & & $100(57,25)$ & \\
\hline & $16: 00-19: 59 h$ & $30(30)$ & & $69(43,50)$ & & $102(62,25)$ & \\
\hline & $20: 00-23: 59 \mathrm{~h}$ & $30(15)$ & & $64(39,25)$ & & $99(52)$ & \\
\hline \multirow{7}{*}{$\begin{array}{l}\text { Día de la } \\
\text { semana }^{c}\end{array}$} & Lunes & $43,15 \pm 49,8$ & \multirow{7}{*}{0,129} & $69,8 \pm 33,2$ & \multirow{7}{*}{0,068} & $112,19 \pm 60,3$ & \multirow{7}{*}{0,826} \\
\hline & Martes & $43,98 \pm 46,07$ & & $73,08 \pm 37,05$ & & $117,06 \pm 58,4$ & \\
\hline & Miércoles & $42,28 \pm 44,9$ & & $72,4 \pm 32,1$ & & $112,74 \pm 54,4$ & \\
\hline & Jueves & $46,55 \pm 49,2$ & & $67,05 \pm 30,6$ & & $112,26 \pm 52,9$ & \\
\hline & Viernes & $43,89 \pm 44,9$ & & $71,3 \pm 30,4$ & & $14,79 \pm 53,02$ & \\
\hline & Sábado & $42,1 \pm 49,09$ & & $75,3 \pm 36,5$ & & $117,04 \pm 57,4$ & \\
\hline & Domingo & $35,83 \pm 34,4$ & & $75,2 \pm 34,6$ & & $111,07 \pm 47,4$ & \\
\hline
\end{tabular}

Tiempo 061: desde que entra la llamada en el centro coordinador de urgencias y actúa el 061 hasta la llegada del paciente al hospital; tiempo de preaviso: desde que ocurre el evento hasta que entra la llamada en el centro coordinador de urgencias; tiempo total: suma de los previos, desde el momento en el que el paciente comienza con el inicio de los síntomas hasta que llega al hospital. ${ }^{a}$ Mediana (rango intercuartílico); ${ }^{b} n$ (\%); ${ }^{c}$ Media \pm desviación estándar.
Tabla V. Tasas de administración de activador del plasminógeno tisular recombinante (rtPA) en ictus isquémicos trasladados por el 061 por variables temporales.

\begin{tabular}{|c|c|c|c|}
\hline & & $\begin{array}{c}\text { Ictus que } \\
\text { recibieron rtPA }\end{array}$ & $p$ \\
\hline \multirow{7}{*}{$\begin{array}{l}\text { Día de } \\
\text { la semana }\end{array}$} & Lunes & $60(23,1 \%)$ & \multirow{7}{*}{0,100} \\
\hline & Martes & $48(18,3 \%)$ & \\
\hline & Miércoles & $55(22,1 \%)$ & \\
\hline & Jueves & $60(23,4 \%)$ & \\
\hline & Viernes & $63(24,5 \%)$ & \\
\hline & Sábado & 75 (30,2\%) & \\
\hline & Domingo & $51(24,4 \%)$ & \\
\hline \multirow{12}{*}{ Mes } & Enero & $31(23,1 \%)$ & \multirow{12}{*}{0,933} \\
\hline & Febrero & $38(25,7 \%)$ & \\
\hline & Marzo & $38(23,3 \%)$ & \\
\hline & Abril & $33(24,1 \%)$ & \\
\hline & Mayo & $43(24,9 \%)$ & \\
\hline & Junio & $27(20,5 \%)$ & \\
\hline & Julio & $28(24,1 \%)$ & \\
\hline & Agosto & $25(22,5 \%)$ & \\
\hline & Septiembre & $34(24,8 \%)$ & \\
\hline & Octubre & $39(25,8 \%)$ & \\
\hline & Noviembre & $44(26,3 \%)$ & \\
\hline & Diciembre & $32(18,6 \%)$ & \\
\hline \multirow{6}{*}{$\begin{array}{l}\text { Horas de } \\
\text { activación }\end{array}$} & $00: 00-03: 59 \mathrm{~h}$ & $15(16,7 \%)$ & \multirow{6}{*}{0,015} \\
\hline & $04: 00-07: 59 \mathrm{~h}$ & $17(16,0 \%)$ & \\
\hline & $08: 00-11: 59$ h & $121(25,5 \%)$ & \\
\hline & $12: 00-15: 59 \mathrm{~h}$ & $108(28,1 \%)$ & \\
\hline & $16: 00-19: 59$ h & $78(24,6 \%)$ & \\
\hline & $20: 00-23: 59 \mathrm{~h}$ & $73(19,8 \%)$ & \\
\hline
\end{tabular}

ictus se producen durante el período de sueño y se descubren al despertar, lo que es un caso particular del ictus de hora de inicio. Se ha descrito que los ictus ocurren con mayor frecuencia durante la ma- 
ñana (06:00-12:00 $\mathrm{h}$ ), particularmente en la primera hora después o antes de despertar, y no existen diferencias en ese sentido entre el primer episodio y las recurrencias $[16,17]$.

Estos ictus de hora de inicio indeterminada se manejan en nuestro medio con un algoritmo diagnóstico y terapéutico diferenciado, en el que los criterios de neuroimagen pesan más y la trombectomía, con su mayor ventana terapéutica, se considera habitualmente la primera opción. Esto debe tenerse en cuenta al analizar el porcentaje de fibrinólisis intravenosas en este primer tramo horario, que sin embargo es el que presenta un mayor porcentaje de aplicación de tratamiento entre las 08:00 y 11:59 h. $\mathrm{El}$ acúmulo de casos al despertar se refleja lógicamente en el número de activaciones del 061 ARAGÓN y también en el número total de fibrinólisis intravenosas, pero creemos que, salvo la mencionada salvedad del peso de los casos de ictus al despertar en la fibrinólisis intravenosa, no debería haber diferencias en el porcentaje de casos efectivamente fibrinolisados en cada tramo horario. No obstante, sí se observan diferencias en el porcentaje de casos tratados con rtPA, que es menor entre las 20:00 y 23:59 h y entre las 04:00 y 07:59 h, lo que sugiere que son factores no dependientes del 061 los determinantes de esas diferencias.

Este estudio viene evidentemente limitado por las variables que recoge el 061 ARAGÓN, que nos parecen insuficientes para la correcta monitorización del proceso asistencial extrahospitalario y consideramos que se deberían revisar. En ese sentido, creemos que sería importante definir un conjunto básico de indicadores que se deberían compartir por todos los servicios de emergencias del Estado, que posibilitara la comparación y la identificación de áreas de mejora y de buenas prácticas. Este conjunto de indicadores debería incluir al menos el número de códigos ictus atendidos, el tiempo desde la activación al lugar de la atención, el tiempo desde la primera asistencia hasta la llegada el hospital, el porcentaje de fibrinólisis intravenosa, el tiempo total desde el evento hasta el inicio de la fibrinólisis intravenosa, el diagnóstico final en los casos en los que se descartó un ictus y la escala de Rankin previa. Los datos de los que disponemos son limitados en lo que hace referencia a la fibrinólisis intravenosa, pues sólo disponemos del total anual y no de otras variables que podrían ser de interés, como la edad, el sexo o el tiempo hasta la fibrinólisis, o los resultados en términos de mortalidad e independencia. La falta de integración de los sistemas de información entre los diferentes agentes (gerencia del 061, hospitales) era, en el período de estudio, una tarea pendiente, aunque ya se han dado pasos en ese sentido.

La investigación sobre el desempeño de los servicios de emergencias en el ictus, como patología dependiente del tiempo [18], es un campo de investigación operativa de gran importancia e interés, y puede centrarse en la mejora de tiempos y en la implantación efectiva de las medidas recomendadas [19]. Es llamativa la escasez de estudios publicados al respecto en nuestro país, y en ese sentido creemos que nuestra aportación cobra más valor al facilitar la comparación con otros grupos. Es de destacar el trabajo realizado en estos temas de organización y tiempos en la asistencia desde Cataluña [2024], así como otras aportaciones y experiencias en Madrid [25], Asturias [26,27] o Murcia [12].

En resumen, sólo el 9,3\% (media interanual) de los pacientes usó el 061, y en el 55,3\% de los pacientes el tiempo 061 fue mayor de 60 minutos. Los pacientes que fueron trasladados por el 061 Aragón recibieron fibrinólisis intravenosa en un porcentaje claramente mayor. La distribución por días de la semana y por meses no mostró diferencias en la activación del código ictus. Sin embargo, hay diferencias en el porcentaje de pacientes que reciben fibrinólisis intravenosa por tramos horarios, y la frecuencia de activaciones del código ictus es mayor en las horas laborables habituales (08:00-12:00 h). Esto no se explica por los tiempos extrahospitalarios, lo que sugiere que, al menos en Aragón, otros factores distintos a los tiempos de atención por el 061, esto es, dependientes de la organización de los hospitales y muy especialmente de sus servicios de urgencias, son determinantes en la aplicación o no del tratamiento fibrinolítico en el ictus agudo.

\section{Bibliografía}

1. Gobierno de Aragón. SALUD. 061 ARAGÓN. URL: http:// www.aragon.es/DepartamentosOrganismosPublicos/OOAA/ ServicioAragonesSalud/AreasTematicas/SectoresSanitarios Centros/ci.09_aragon_urgencias.detalleDepartamento?chan nelSelected=b729dd2a38e2b210VgnVCM100000450a15acR CRD. [22.10.2018].

2. Matías-Guiu Guía J. Ministerio de Sanidad y Política Social. Estrategia en ictus del sistema nacional de salud. 2009. URL: http://www.mscbs.gob.es/organizacion/sns/planCalidadSNS/ docs/EstrategiaIctusSNS.pdf. [22.10.2018].

3. Gómez-Angelats E, Bragulat E, Obach-Baurier V, GómezChoco M, Sánchez M, Miró O. Resultados alcanzados con la puesta en marcha del circuito 'Código Ictus' en un gran hospital: papel de urgencias y análisis de la curva de aprendizaje. Emergencias 2009; 21: 105-13.

4. Marta-Moreno J, Bestué-Cardiel M, Giménez-Muñoz A, Palacín-Larroy M. Programa de Atención al Ictus en Aragón (PAIA). Estrategia del cambio y resultados en el período 2009-2014. Neurología 2018; 33: 301-12.

5. Gobierno de Aragón. Plan de atención al ictus en Aragón 2019. Consejería de Sanidad. URL: https://www.aragon.es/estaticos/ GobiernoAragon/Departamentos/SanidadBienestarSocial 
Familia/Sanidad/Documentos/Programa_Ictus_actualizacion 2019.pdf. [06.06.2019].

6. Giménez-Muñoz A, Palacín-Larroy M, Bestué-Cardiel M, Marta-Moreno J. El audit como herramienta de mejora continu en el Plan de Atención al Ictus en Aragón. Rev Neurol 2016; 63: 49-57.

7. Instituto Nacional de Estadística. España en cifras 2019. URL https://www.ine.es/prodyser/espa_cifras/2019/. [01.06.2019].

8. Saver JL, Fonarow GC, Smith EE, Reeves MJ, Grau-Sepúlveda MV, Pan W, et al. Time to treatment with intravenous tissue plasminogen activator and outcome from acute ischemic stroke. JAMA 2013; 309: 2480-8.

9. Instituto Aragonés de Estadística. Datos del padrón municipal de habitantes a 1 de enero de 2017. URL: http://www.aragon es/DepartamentosOrganismosPublicos/Institutos/Instituto AragonesEstadistica/AreasTematicas/02 Demografia Y Poblacion/01_CifrasPoblacion_Y_Censos/01_Padron/ci.01 Cifras_oficiales_poblacion.detalleDepartamento?channelSe ected $=0$. [20.01.2019].

10. Instituto Nacional de Estadística. Índice de envejecimiento por comunidad autónoma. URL: http://www.ine.es/jaxiT3/ Datos.htm?t=1452. [20.01.2019].

11. Iglesias-Mohedano A.M, García-Pastor A, García-Arratibel A Sobrino-García P, Díaz-Otero F, Romero-Delgado F, et al. Identificación de los factores que influyen en el retraso intrahospitalario del inicio de trombólisis intravenosa en el ictus agudo en un hospital terciario. Rev Neurol 2016; 31: 452-8.

12. Palazón-Cabanes B, López-Picazo Ferrer JJ, Morales-Ortiz A, Tomás-García N. ¿Por qué se retrasa el tratamiento de reperfusión en pacientes con código ictus? Un análisis cualitativo. Revista de Calidad Asistencial 2016; 31: 347-55.

13. Avellaneda-Gómez C, Rodríguez-Campello A, GiraltSteinhauer E, Gómez-González A, Serra-Martínez M De Ceballos-Cerrajería P, et al. Estudio descriptivo de los stroke mimics después de un estudio neurovascular completo. Neurología 2019; 34: 7-13.

14. Okano Y, Ishimatsu K, Kato Y, Yamaga J, Kuwahara K, Okumoto K, et al. Clinical features of stroke mimics in the emergency department. Acute Med Surg 2018; 5: 241-8.

15. Tobin WO, Hentz JG, Bobrow BJ, Demaerschalk BM. Identification of stroke mimics in the emergency department setting. J Brain Dis 2009; 1: 19-22.

16. Mackey J, Kleindorfer D, Sucharew H, Moomaw CJ, Kissela BM Alwell K. Population-based study of wake-up strokes. Neurology 2011; 76: 1662-7.
17. Ferre A, Ribó M, Rodríguez-Luna D, Romero O, Sampol G, Molina CA, et al. Los ictus y su relación con el sueño y los trastornos del sueño. Neurología 2013; 28: 103-18.

18. Jiménez-Fàbrega X, Espila-Etxeberria J.L, Gallardo-Mena J. Códigos de activación: pasado, presente y futuro en España. Servicio Navarro de Salud-Osansubidea. Pamplona, España. Emergencias 2011; 23: 311-8.

19. Kobayashi A, Czlonkowska A, Ford GA, Fonseca AC, Luijckx GJ, Korv J, et al. European Academy of Neurology and European Stroke Organization consensus statement and practical guidance for pre-hospital management of stroke. Eur J Neurol 2018; 25: 425-33.

20. Abilleira S, Lucente G, Ribera A, Permanyer-Miralda G, Gallofré M. Patient related features associated with a delay in seeking care after stroke. Cataluña. Eur J Neurol 2011; 18: 850-6.

21. Carrera D, Gorchs M, Querol M, Abilleira S, Ribó M, Millán M, et al. Revalidation of the Race Scale after its regional implementation in Catalonia: a triage tool for large vessel occlusion. Catalan Stroke Code and Reperfusion Consortium (Cat-SCR). J Neurointerv Surg 2018; Dec 22. [Epub ahead of print].

22. Pérez de la Ossa N, Ribó M, Jiménez X, Abilleira S. Prehospital scales to identify patients with large vessel occlusion: it is time for action. Stroke 2016; 47: 2877-8.

23. Pérez de la Ossa N, Carrera D, Gorchs M, Querol M, Millán M, Gomis M, et al. Design and validation of a prehospital stroke scale to predict large arterial occlusion: the rapid arterial occlusion evaluation scale. Stroke 2014; 45: 87-91.

24. Abilleira S, Dávalos A, Chamorro A, Álvarez-Sabín J, Ribera A, Gallofré M. Outcomes of intravenous thrombolysis after dissemination of the stroke code and designation of new referral hospitals in Catalonia: the Catalan Stroke Code and Thrombolysis (Cat-SCT) Monitored Study. Stroke 2011; 42: 2001-6.

25. Alonso de Leciñana-Cases M, Gil-Núñez A, Díez-Tejedor E. Relevance of stroke code, stroke unit and stroke networks in organization of acute stroke care. The Madrid acute stroke care program. Cerebrovasc Dis 2009; 27 (Suppl 1): S140-7.

26. García-Cabo C, Benavente L, Martínez-Ramos J, PérezÁlvarez A, Trigo A, Calleja S. Análisis del primer año del nuevo protocolo de código ictus en Asturias. Experiencia de un único centro. Asturias. Neurologia 2018; 33: 92-7.

27. Benavente L, Villanueva MJ, Vega P, Casado I, Vidal JA Castaño B, et al. El código ictus de Asturias. Neurología 2016; 31: $143-8$.

\section{Characteristics of the stroke alert process attended by 061 ARAGON assistance units from 2010 to 2016. Factors influencing times of response and access to fibrinolytic treatment}

Aim. To study the response times of stroke code assistance by care units of 061 ARAGON, analyzing factors involved and their relationship with availability to fibrinolytic treatment in the hyperacute phase.

Patients and methods. Transversal descriptive study on outpatient care from the registry of cases attended by 061 ARAGÓN health care units to patients with stroke code during the period 2010-2016.

Results. A total of 1743 patients were attended ( $54.6 \%$ males), with a mean age of $72.83 \pm 13.1$ years. There was a higher number of strokes attended in 2015 and 2016 (372 and 366 respectively), compared to the average of 201 strokes per year in the rest of the years. 27,2\% of patients were treated between 08:00-11:59 $\mathrm{h}$, the most frequent time interval. Regarding the time, it took to take care of the patient, the average was $71.93 \pm 33.64$ minutes with a longer response time in Teruel. When influence of the hour interval on the percentage of cases treated with fibrinolysis was analyzed, a higher rate of fibrinolysis was observed when it was activated between 12:00-15:59 $\mathrm{h}$ (28.1\%).

Conclusions. In 55,3\% of patients, the 061 response time was greater than 60 minutes, but this time were not conditioned by the activation time. However, there were differences in the percentage of cases fibrinolysed in the different time intervals, suggesting that factors other than the response time of 061 influence the indication of fibrinolytic treatment.

Key words. Fibrinolysis. Ischemic stroke. Outpatient care. Response time. Stroke code. Stroke mimic 